\title{
Surface tension of aqueous binary mixtures under the supercooled conditions - Development of the measuring technique and preliminary data for water + lower alcohols
}

\author{
Václav Vinš ${ }^{1,2^{*}}$, Jiří Hykl ${ }^{1}$, Zbyněk Nikl ${ }^{1}$, Miroslav Čenský ${ }^{1}$, and Jan Hrubý ${ }^{1}$ \\ ${ }^{1}$ Institute of Thermomechanics of the CAS, Dolejškova 1402/5, Prague 8, 182 00, Czech Republic \\ ${ }^{2}$ Škoda Auto, tř. Václava Klementa 869, Mladá Boleslav, 293 01, Czech Republic
}

\begin{abstract}
An experimental apparatus originally developed for the measurement of surface tension of supercooled water was modified such that it allows for measurement of binary aqueous mixtures. The measuring principle based on the capillary rise technique is similar to that employed in the previous measurements with pure water [J. Hrubý et al., J. Phys. Chem. Lett. 5 (2014) 425 and V. Vinš et al., J. Phys. Chem. B 119 (2015) 5567]. The temperature dependence of the surface tension is determined from the measured height of the liquid column elevated in a vertical capillary tube with an inner diameter of $0.32 \mathrm{~mm}$. The aqueous liquid rises to a height of $60 \mathrm{~mm}$ to $95 \mathrm{~mm}$ depending on the solute concentration and the temperature. Preliminary tests performed with the binary mixtures of water with methanol, ethanol, and $n$-propanol at various concentrations and temperatures down to $-28{ }^{\circ} \mathrm{C}$ approved functionality of the measuring technique. Some difficulties, such as influence of impurities on the liquid column elevation or formation of bubbles in the liquid column, were observed. The experimental apparatus is further being modified in order to obtain more accurate data for various aqueous mixtures.
\end{abstract}

\section{Introduction}

This study is part of a long-term research of our group focussing on the experimental investigation of thermophysical properties of water and aqueous systems under the supercooled conditions, i.e. on the properties of the metastable liquid aqueous phase at temperatures below the equilibrium freezing temperature. Water shows several anomalies at low temperatures, e.g., the commonly known maximum in density at $+4{ }^{\circ} \mathrm{C}$ or a sharp increase of isobaric heat capacity and isothermal compressibility at temperatures approaching $-38{ }^{\circ} \mathrm{C}[1,2]$, i.e. a macroscopic supercooling limit of homogeneous freezing. The surface tension was expected to show another anomaly referred as the SIP (second inflection point) anomaly in its temperature trend at temperatures close to $-8^{\circ} \mathrm{C}$. However, the existence of SIP was recently refuted down to $-26^{\circ} \mathrm{C}$ by our experiments [3-5].

In this work, the experimental apparatus and the measuring technique used for the measurement of the surface tension of pure supercooled water [3-7] was modified such that it allows for investigation of aqueous binary mixtures. The new accurate data for the surface tension of aqueous systems, such as mixtures with alcohols or salts, under the supercooled conditions are important for scientific communities focused on the atmospheric and oceanographic research $[8,9]$ and on the investigation of anomalous behaviour of water systems at low temperatures [1,2]. Functionality of the new measuring technique was successfully tested by initial measurements of water mixed with several short-chain alcohols; namely with methanol, ethanol, and $n$-propanol.

\section{Experimental Apparatus}

The experimental apparatus for the measurement of surface tension of pure water under the supercooled conditions was described in previous studies $[3,4,7]$ in detail. The measuring principle is based on a modified capillary-rise technique, when the upper part of a vertical capillary tube is positioned in a temperature-controlled chamber. The capillary tube is located in the chamber such that the temperature of the liquid meniscus inside the capillary tube could be varied. The surface tension $\sigma$ and eventually its temperature trend can be determined from the measured height $h$ of the liquid column elevated in the capillary tube of a known inner diameter $d$.

$$
\sigma=\frac{h \rho g d}{4 \cos \theta}
$$

In equation (1), $\rho$ stands for the density of liquid, $g$ is the gravity, and $\theta$ denotes the contact angle between the liquid and the capillary tube wall. Basic principle of the measuring technique is depicted in Figure 1. Height $h$, i.e. the distance between the liquid meniscus inside the capillary tube and the liquid level inside the lower container is measured by using a digital camera installed on a high-precision height gauge. The standard uncertainty of the measured height was below $0.012 \mathrm{~mm}$.

\footnotetext{
*Corresponding author: vins.vaclav@seznam.cz
} 


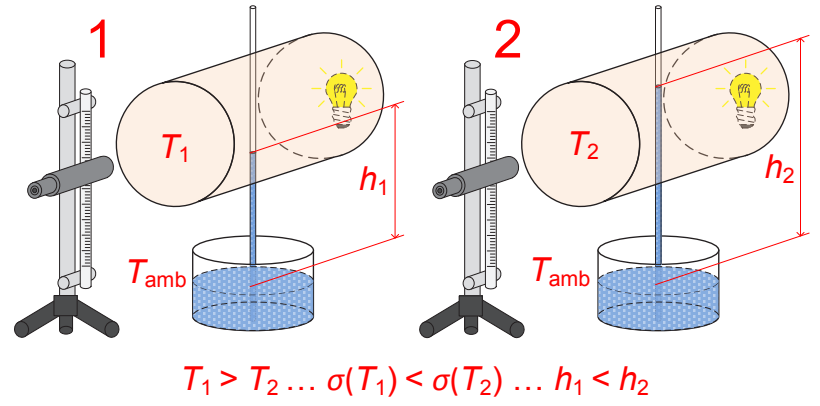

Figure 1. Scheme of the experimental apparatus with the vertical capillary tube partially placed inside a temperaturecontrolled chamber. Case 1 corresponds to the reference measurement, e.g., at $+15^{\circ} \mathrm{C}$, and case 2 is performed at the target temperature.

\section{Description of Measurement}

The mixture samples were prepared by the weighing method using analytical scale Mettler Toledo XSE $205 D U$ with accuracy better than $0.1 \mathrm{mg}$. The samples were mixed using an ultrasonic homogenizer in order to assure homogeneous distribution of the composition. The uncertainty of the mass fraction of the binary samples was better than 0.0001 . Ultrapure water, prepared from the serially connected purification devices reverse osmosis unit Watrex Rowapur and Neptune Analytical, was used both for the capillary tube cleaning and for the preparation of measured samples. The ultrapure water had a constant resistivity of $18.2 \mathrm{M} \Omega$, total organic carbon content below $5 \mathrm{ppb}$ and was free of particles larger than $0.2 \mu \mathrm{m}$. A sample of methanol was purchased from Lach-Ner and had declared purity of $99.99 \%$. Sample of absolute ethanol was purchased from Penta Chemicals and had declared purity of $99.99 \%$. Sample of $n$-propanol was purchased from Penta Chemicals and had declared purity of $99.94 \%$.

Temperature inside the temperature-controlled chamber was measured with two in-house calibrated resistive thermometers Pt100 located close to the capillary tube. The standard uncertainty of the measured temperature was better than $0.040^{\circ} \mathrm{C}$. The capillary tube was repeatedly cleaned with chromosulfuric acid and ultrapure water. The upper end of the capillary tube was connected to the helium distribution setup [6], which allowed flushing of the capillary tube with pure helium. All measurements were carried out with descending meniscus by applying a small underpressure above the liquid meniscus using a syringe installed at the helium distribution setup.

The surface tension was measured with a technique similar to that employed in the previous studies with pure water $[3,4]$ referred as the height measurement. The main difference was that two independent measurements with pure water were conducted before and after the measurements with an aqueous mixture. The reference measurements with pure water at a constant temperature of $+15^{\circ} \mathrm{C}$ were used for the evaluation of the inner diameter of the capillary tube. The inner diameter was determined from the reference measurements and the international standard for the surface tension of ordinary water [10] approved by IAPWS [11]. Consequently, all measurements presented in this work are relative and related to the surface tension of pure water at $+15^{\circ} \mathrm{C}$. The magnitude of the inner diameter of all capillary tubes employed in this study lied in the range from $0.3220 \mathrm{~mm}$ to $0.3230 \mathrm{~mm}$ with a standard uncertainty better than $0.0020 \mathrm{~mm}$.

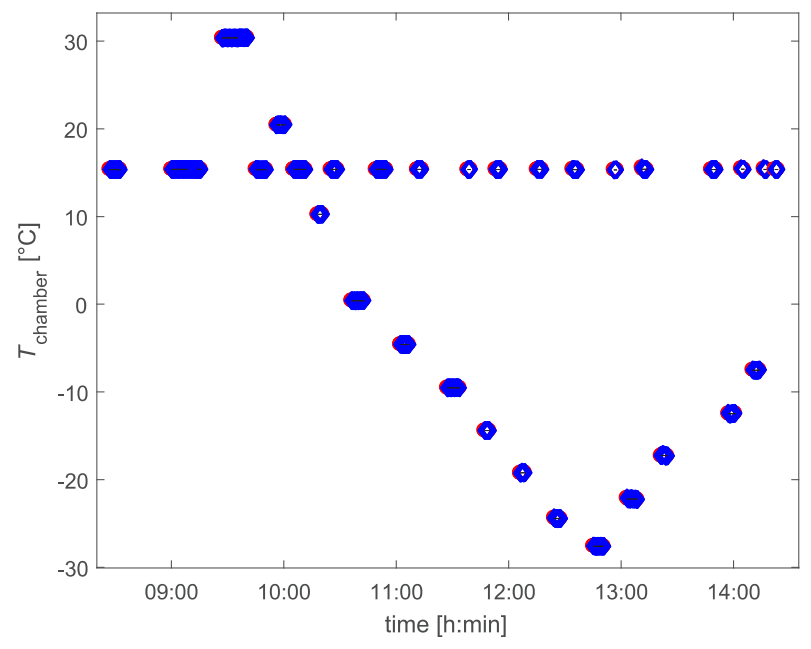

Figure 2. Temperature variation during one experimental run. Measurement with $10 \%$ mass fraction of methanol.

Figure 2 shows variation of temperature inside the temperature-controlled chamber over time. It shall be noted that only the stabilized conditions are shown. The measurement at the desired temperature was every time followed by the measurement at the reference temperature of $+15^{\circ} \mathrm{C}$. The reference measurements helped to detect possible negative effects during the experiments, such as presence of impurities on the capillary tube wall, degradation of the sample, i.e. variation of its composition, etc. We note that no variation of the sample composition over the time of measurement was detected during our experiments.

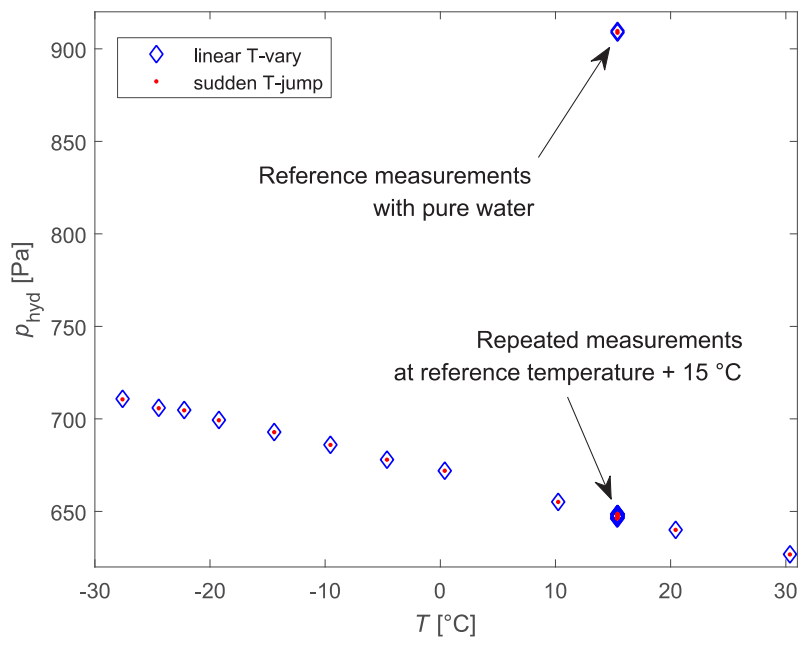

Figure 3. Hydrostatic pressure of the liquid column inside the capillary tube measured for the aqueous binary mixture with $15 \%$ mass fraction of methanol. Comparison of the linear temperature change over the transient region of $3 \mathrm{~mm}$ height and the sudden temperature jump. 
Hydrostatic pressure of the liquid column elevated in the capillary tube given as follows

$$
p_{\text {hyd }}=h \rho g
$$

is depicted in Figure 3 for a mixture of water with a $15 \%$ mass fraction of methanol. Due to a significant decrease in the surface tension caused by the presence of methanol, the hydrostatic pressure at $+15^{\circ} \mathrm{C}$ dropped from $909 \mathrm{~Pa}$ corresponding to the pure water sample down to $648 \mathrm{~Pa}$ in case of the mixed sample.

Both the measurements with mixed sample at the reference temperature of $+15^{\circ} \mathrm{C}$ performed throughout the experimental run and the reference measurements with pure water conducted before and after the mixture measurement show considerably good internal consistency as can be seen in Figure 3. No negative effects during the measurement were observed in this case.

Nevertheless, not every experimental test could be treated as successful. Measurements under the metastable conditions are rather difficult as the purity of the sample, the cleanness of the experimental apparatus and above all the time scale of the measurement matter a lot. Figure 4 shows an interesting phenomenon of an unsuccessful measurement, when small bubbles appeared in the liquid column elevated in the capillary tube. Three stages of the gradual bubble formation can be seen in the figure. Possible reason for the bubble formation could be a presence of impurities or small cracks on the capillary tube inner surface avoiding continuous movement of the liquid column together with an inhomogeneous composition of the mixed sample along the capillary tube length.

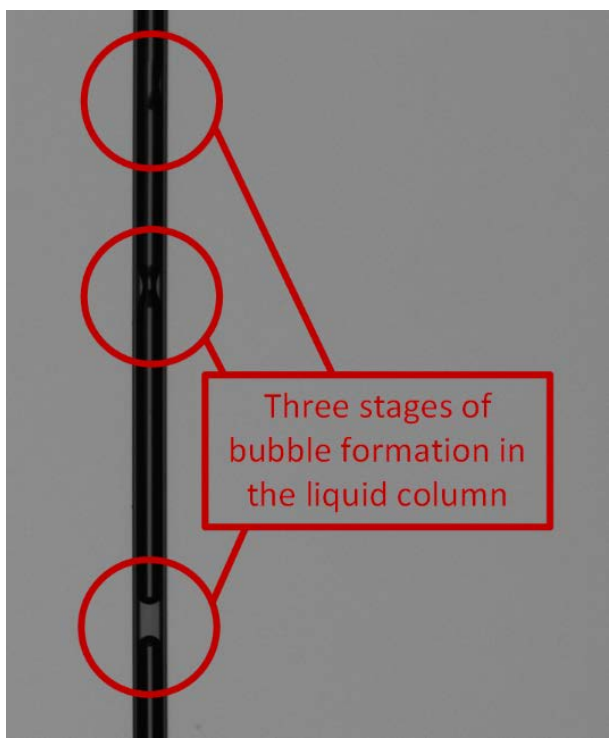

Figure 4. Photo of the capillary tube inside the temperaturecontrolled chamber with three stages of the bubble formation within the liquid column of water and lower alcohol mixture.

\section{Results}

Functionality of the new measuring technique was investigated on the measurements of aqueous binary mixtures with methanol, ethanol, and $n$-propanol. The data were collected in the temperature range from
$-28{ }^{\circ} \mathrm{C}$ to $+31{ }^{\circ} \mathrm{C}$, i.e. both in the stable region and under the supercooled metastable conditions. Figure 5 shows preliminary testing measurements for two mixtures with ethanol and $n$-propanol with mass fraction of alcohol of 5 $\%$ in both cases. The experimental data are compared with the IAPWS correlation [11] for the surface tension of pure water and the water-lower alcohol experimental parametrization suggested by Němec [12]. At the reference temperature of $+15^{\circ} \mathrm{C}$, adding $5 \%$ of ethanol resulted in a decrease of the surface tension by $16.5 \mathrm{mN} \mathrm{m}^{-1}$ compared to pure water. In case of $n$ propanol the decrease became even more significant of around $26.5 \mathrm{mN} \mathrm{m}^{-1}$. For both mixtures, the experimental data show quite good agreement with the theoretical parametrization at temperatures above $+15^{\circ} \mathrm{C}$. However at sub-zero temperatures, the experimental data deviate from the correlation. The deviation is particularly significant in case of $n$-propanol at temperatures below $5^{\circ} \mathrm{C}$. The continuous increase of the surface tension with decreasing temperature is qualitatively similar to the SIP anomaly of pure water detected at temperature around $8{ }^{\circ} \mathrm{C}[3,13]$. Nevertheless, the presented data are only preliminary and further experimental investigation of this phenomenon is needed. Unfortunately this represents an uneasy task due to some difficulties by the measurements with ethanol and $n$-propanol, such as formation of bubbles within the liquid column shown in Figure 4.

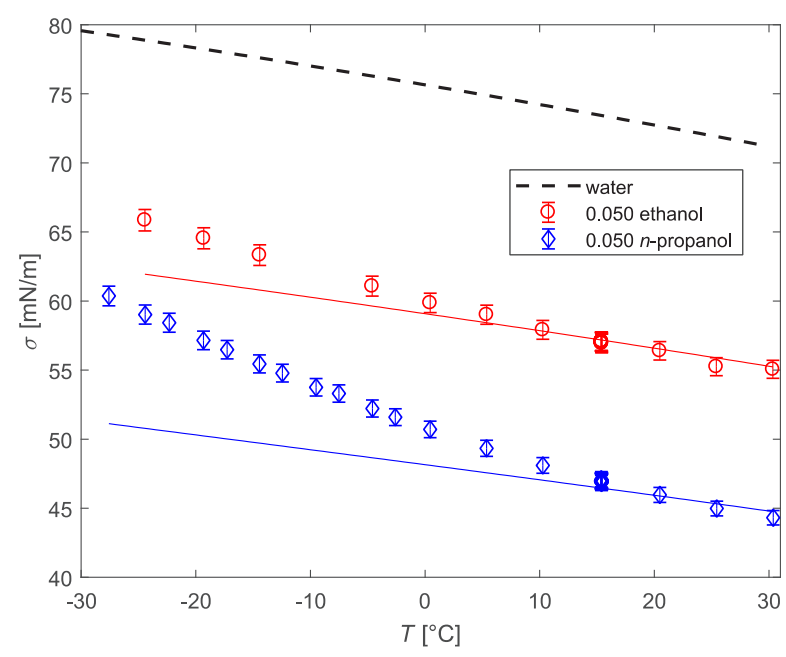

Figure 5. Results of preliminary testing measurements of the surface tension of water + ethanol and water $+n$-propanol. The mass fraction of alcohol was $5 \%$ in both cases. Black dashed line represents IAPWS correlation [11] for pure water extrapolated below $0.01{ }^{\circ} \mathrm{C}$. Red and blue solid lines are predictions of the water-short chain alcohol correlation [12].

The water-lower alcohol model combines the surface tension of pure water obtained from the IAPWS correlation [11] and the surface tension of pure alcohols predicted using correlations by Mulero et al. [16]. The values for pure components are combined using ConnorsWright mixing formula [17]. In case of water + ethanol mixture, the correlation by Wilemski [18] was used.

In the data analysis, density of water + lower alcohol systems required in equations (1) and (2) was predicted with the Redlich-Kister type of parametrization, in which the densities of pure water and pure alcohols were 
calculated using the reference equation of state IAPWS95 [19] and the parametrizations from the LandoltBörnstein series [20], respectively. For more details see ref. [12]. A constant contact angle $\theta=0.5^{\circ}$ was considered for all measurements with mixed samples. In the evaluation of the capillary tube inner diameter, a constant contact angle of $3 \pm 1^{\circ}$ was considered for pure water and fused silica in case of the descending meniscus $[14,15]$. We note that the influence of the contact angle is minor compared to other quantities in equation (1).

Figure 6 shows data for the surface tension of water + methanol binary systems at five different mass fractions of methanol ranging from 0.03 to 0.20 . The data are compared to the IAPWS correlation [11] for pure water and the water + lower-alcohol mixture correlation [12]. As can be seen the data agree quite well with the mixture model over the entire temperature range from $-28{ }^{\circ} \mathrm{C}$ to $+31^{\circ} \mathrm{C}$. The only considerable difference can be seen in case of the lowest concentration of 0.03 . Unlike in case of mixtures with ethanol and $n$-propanol depicted in Figure 5 , no significant growth in the surface tension at low temperatures was observed for the water + methanol system. The data have almost constant slope in the experimental temperature range.

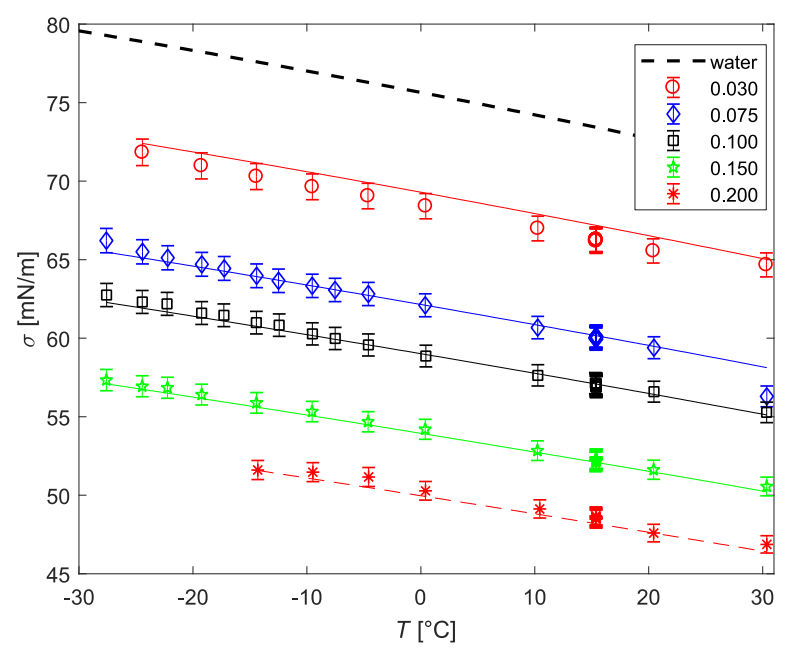

Figure 6. Surface tension of water + methanol including supercooled region. Values in the legend correspond to mass fraction of methanol. Black dashed line represents IAPWS [11] correlation for the surface tension of ordinary water extrapolated below $0.01{ }^{\circ} \mathrm{C}$, other lines are predictions of experimental correlation [12].

As the presented data have preliminary character, the standard uncertainty of the resulting surface tension is estimated relatively large $u(\sigma) \leq 0.80 \mathrm{mN} \mathrm{m}^{-1}$ compared to pure water measurements with the maximum uncertainty of around $0.40 \mathrm{mN} \mathrm{m}^{-1}$ [3-5]. Main sources of the uncertainty are coming from the uncertainty of the IAPWS correlation [11] for the surface tension of pure water at the reference temperature of $+15^{\circ} \mathrm{C}$ $u\left(\sigma_{\text {IAPWS }}\right)=0.37 \mathrm{mN} \mathrm{m}^{-1}$, which is used in the determination of the capillary tube inner diameter, and from the density of the binary mixture, which is considered quite large $u_{\mathrm{r}}(\rho)=1.0 \%$ as it contains also the uncertainty of the mixture composition.

\section{Conclusions}

The experimental apparatus for the measurement of surface tension of water under the metastable supercooled conditions was successfully modified in order to investigate aqueous binary mixtures. The preliminary measurements with water + short-chain alcohols proved functionality of the new measuring technique. Data for the binary system water + methanol at mass fractions of methanol varying from 0.03 to 0.20 show good agreement with the theoretical parametrization [12] over the entire experimental temperature range from $-28{ }^{\circ} \mathrm{C}$ to $+31{ }^{\circ} \mathrm{C}$. On the other hand, preliminary measurements of binary aqueous systems with ethanol and $n$-propanol deviate from the model, as a significant increase in the surface tension was detected at sub-zero temperatures. The experimental temperature trend similar to the SIP anomaly of the pure water [3] is particularly pronounced in case of $n$-propanol. Further experimental investigation is required in order to clarify this phenomenon.

We note that the apparatus is further being modified in order to improve the accuracy of the measured data. The experimental setup is currently being used for the measurements of surface tension of water + sodium chloride and the supercooled seawater.

\section{Acknowledgement}

The study was supported by the Czech Science Foundation grant GJ15-07129Y and by the institutional support RVO:61388998 from IT CAS.

\section{References}

1. P.H. Poole, F. Sciortino, U. Essmann, H.E. Stanley, Nature 360, 324 (1992)

2. O. Mishima, H.E. Stanley, Nature 396, 329 (1998)

3. J. Hrubý, V. Vinš, R. Mareš, J. Hykl, J. Kalová, J. Phys. Chem. Lett. 5, 425 (2014)

4. V. Vinš, M. Fransen, J. Hykl, J. Hrubý, J. Phys. Chem. B 119, 5567 (2015)

5. V. Vinš, J. Hošek, J. Hykl, J. Hrubý, J. Chem. Eng. Data 62, 3823 (2017)

6. V. Vinš, J. Hrubý, J. Hykl, J. Blaha, B. Šmíd, EPJ Web Conf. 45, 01094 (2013)

7. V. Vinš, J. Hošek, J. Hykl, J. Hrubý, EPJ Web Conf. 92, 02108 (2015)

8. Y. Viisanen, R. Strey, H. Reiss, J. Chem. Phys. 99, 4680 (1993)

9. M.A.L.J. Fransen, E. Sachteleben, J. Hrubý, D.M.J. Smeulders, Exp. Fluids 55, 1780-1 (2014)

10. N.B. Vargaftik, B.N. Volkov, L.D. Voljak. J. Phys. Chem. Ref. Data 12, 817 (1983)

11. IAPWS revised release on surface tension of ordinary water substance. 2014; http://www.iapws.org

12. T. Němec, Institute of Thermomechanics of the CAS, Prague, Czech Republic, private communication

13. P.T. Hacker, NACA TN 2510 (1951) 
14. V.G. Stepanov, L.D. Volyak, Y.V. Tarlakov, J. Eng. Phys. Thermophys. 32, 646 (1977). Translated from Inzhenerno-Fizicheskii Zhurnal 32, 1000 (1977)

15. L.D. Volyak, V.G. Stepanov, Y.V. Tarlakov, Zh. Fiz. Khim. 49, 2931 (1975)

16. A. Mulero, I. Cachadina, and E. L. Sanjuán, J. Phys. Chem. Ref. Data 44, 033104 (2015)

17. C.-W. Chang, T.-L. Hsiung, C.-P. Lui, C.-H. Tu, Fluid Phase Equilib. 389, 28 (2015)

18. G. Wilemski, J. Chem. Phys. 91, 2492 (1987).

19. W. Wagner, A. Pruß, J. Phys. Chem. Ref. Data 31, 387 (2002).

20. M. Frenkel, X. Hong, R. C. Wilhoit, K. R. Hall, Densities of Alcohols, edited by K. R. Hall and K. N. Marsh, Landolt-Börnstein, New Series, Vol. IV/8G (Springer, 1998). 